\title{
QUATERNION MATRIX SINGULAR VALUE DECOMPOSITION AND ITS APPLICATIONS FOR COLOR IMAGE PROCESSING
}

\author{
Soo-Chang Pei Ja-Han Chang and Jian-Jiun Ding \\ Department of Electrical Engineering, National Taiwan University, Taipei, Taiwan 10617, R. O. C. \\ E-mail: pei@cc.ce.ntu.cdu.tw Fax: 886-2-23671909
}

\begin{abstract}
In this paper, we first discuss the singular value decomposition (SVD) of a quaternion matrix and propose an algorithm to calculate the SVD of a quaternion matrix using its equivalent complex matrix. The singular values of a quaternion matrix are still real and positive, but the two unitary matrices are quaternion matrices with quaternion entries.

Then, applications for color image processing by the SVD of a quaternion matrix are given. Since a quatemion matrix can represent a color image, so we can use the SVD of a quaternion matrix to decompose a color image. Therefore, many useful image processing methods by SVD, such as eigen-images, image compression, image enhancement and denoise, can be extended to color image processing without separating the color image into three channel images.
\end{abstract}

\section{INTRODUCTION}

The well-known concept of quatemions was introduced by Hamilton in 1843 [1]. Quaternions are the generalization of complex numbers. A quaternion has 4 components, i.e., one real part and three imaginary parts:

$$
q=q_{\mathrm{r}}+q_{\mathrm{s}} \cdot i+q_{3} \cdot j+q_{\mathrm{k}} \cdot k
$$

and $i, j, k$ obey the rules as below:

$$
\begin{gathered}
i^{2}=j^{2}=k^{2}=-1, \quad i \cdot j=k, \quad j \cdot k=i, \quad k \cdot i=j, \\
j \cdot i=-k, \quad k \cdot j=-i, \quad i \cdot k=-j .
\end{gathered}
$$

Besides, by negating the three imaginary parts, every quaternion $q$ has its conjugate, $q^{*}=q_{\mathrm{r}}-i q_{\mathrm{i}}-j q_{\mathrm{j}}-k q_{\mathrm{k}}$. From (2), we can find that the multiplication rule of quaternions is not commutative.

In this paper, we focus on the quaternion matrices, so the detailed introduction of quaternions is not discussed here. This information can be found in [1].

Until now, some papers on the quaternion matrices have been published. In 1947, H. C. Lee gave a discussion about the calculation of the eigen-values of a quaternion matrix [2]. The eigen-values of a quaternion matrix can be calculated by the eigen-values of an equivalent complex matrix. In $1950, \mathrm{~J} . \mathrm{L}$. Brenner gave some theorems for the quaternion matrix [3]. Brenner has shown that every quaternion matrix can be transformed into triangular form and has a characteristic root. In 1989, A. Bunse-Gerstner, R. Byers and V. Mehrmann developed a quaternion $Q R$ algorithm [4]. Every quaternion matrix can be decomposed into the multiplication of 2 matrices, $Q$ and $R$, where $Q$ is a unitary matrix and $R$ is a upper triangular matrix. In 1997, F. Zhang gave a detailed discussion about the quaternion matrix [5]. Using the same concept as $\mathrm{H}$. C. Lee, Zhang proved that the existence of the polar decomposition of any quaternion matrix and this proof process can be used for the existence of SVD.

In this paper, we use the same concept as H. C. Lee and F. Zhang to calculate the eigen-values of the quaternion matrix and develop an algorithm to compute the SVD of a quaternion matrix by means of the SVD of its equivalent complex matrix.

The rest of this paper is organized as follows. In Sec. 2, we discuss the eigen-values and eigen-vectors of a quaternion matrix. An equivalent complex matrix is useful to calculate these elements. In Sec. 3, we give an algorithm to compute the SVD of a quaternion matrix by the SVD of its equivalent complex matrix. Then, applications of the SVD of a quaternion matrix for color image processing are given in Sec. 4. Many useful image processing methods by SVD can be extended to color image processing without separating the color image into three channel images. Finally, conclusions are made in Sec. 5.

Besides, we will use the following notations:

- $Q_{(\mathrm{q})}, Q_{(\mathrm{c})}$ : A quaternion matrix and a complex matrix.

- $\vec{x}_{(q)}, \vec{x}_{(c)}$ : A quaternion vector and a complex vector.

- $\mathrm{C}^{+}$: The set of complex numbers with nonnegative imaginary part.

- $Q_{e(c)}$ : The equivalent complex matrix of a quaternion matrix $Q_{(q)}$

\section{EIGEN-VALUES AND EIGEN-VECTORS OF A QUATERNION MATRIX}

The eigen-values and eigen-vectors of a quaternion matrix have been discussed in $[2,5]$. Here, we will give a brief review.

Definition 1 : Left eigen-values and right eigen-values

Due to the non-commutative multiplication rule of quaternions, the eigen-values of a quaternion matrix have two categories, left eigen-values and right eigen-values, which satisfy the following equations, respectively.

$$
Q_{(q)} \cdot \vec{x}_{(q)}=\lambda \cdot \vec{x}_{(q)} \text { (Left), } Q_{(q)} \cdot \vec{x}_{(q)}=\vec{x}_{(q)} \cdot \lambda \text { (Right) }
$$

In this paper, we only use the right eigen-values and we adopt the name 'eigen-value' to represent the right eigen-value.

\section{Derinition 2 : Eigen-value class}

If $\lambda$ is one eigen-value of a quaternion matrix $Q_{(\mathrm{q})}$, then every element of the set $\Gamma \equiv\left\{q \lambda q^{-1}\right.$ : where $q$ is any unit quaternion with its norm $|q|^{=1\}}$ is also an eigen-value of $Q_{(q)}$. Moreover, this set $\Gamma$ will contains a single eigen-value $\lambda_{c} \in C^{+}$so that this set is called the eigen-value class of $\lambda_{c}$. (The proof can be found in [4][5]) Furthermore, the conjugate of $\lambda_{c}$ also belongs to $\Gamma$. Except for $\lambda_{c}$ and its conjugate, all other elements in $\Gamma$ are quaternion numbers. 
Therefore, the eigen-values of a quaternion matrix are infinite However, the eigen-value classes are finite. A $n^{*} n$ quaternion matrix will have $n$ eigen-values classes, that is, $n$ complex eigen-values with nonnegative imagine part.

In the sequel, we only calculate the complex eigen-values with nonnegative imaginary part $\lambda_{r} \in C^{+}$of a quaternion matrix.

Definition 3 : Equivalent complex matrix of a quaternion matrix Let $Q_{(q)}$ be a $n^{*} n$ quaternion matrix and $Q_{(q)}=\mathrm{A}_{(\mathrm{c})}+\mathrm{B}_{(\mathrm{c})} \mathrm{j}$, where $A_{(c)}$ and $B_{(c)}$ are two complex matrices, then the equivalent complex matrix of $Q_{(\mathrm{q})}$ is

$$
Q_{\mathrm{e}(c)}=\left[\begin{array}{cc}
A_{(c)} & -\bar{B}_{(c)} \\
B_{(c)} & \bar{A}_{(c)}
\end{array}\right]_{2 m \times 2 n}
$$

The relations between the quaternion matrix and its equivalent complex matrix can be found in [5] and are brietly summarized in theorem 1 .

Theorem 1 : Relations between a quaternion matrix and its equivalent complex matrix

Let $Q_{(q)}, Q_{1(q)}$ and $Q_{2(q)}$ be three $n^{*} n$ quaternion matrices, then

(a) If $Q_{\langle q\rangle}=Q_{\langle(q)} \cdot Q_{2(q)}$, then $Q_{e(c)}=Q_{1, e(c)}, Q_{2, e(c)}$.

(b) $Q_{p(c)}^{\square}$ is the equivalent complex matrix of $Q_{(q)}^{\square}$, where the superscript $\mathrm{D}=\mathrm{H}$ or $-1\left({ }^{\mathrm{H}}\right.$ means the hermitian transpose and ${ }^{-1}$ means the inverse of the matrix.)

Therefore, many properties of $Q_{(\mathrm{q})}$ and $Q_{\mathrm{e}(\mathrm{c})}$ are similar. Besides, we can use the eigen-values and eigen-vectors of $Q_{\text {e(c) }}$ to find the eigen-values and eigen-vectors of $Q_{(\mathrm{q})}$.

Theorem 2 : Eigen-values and eigen-vectors of a quaternion matrix and its equivalent complex matrix. [5]

Let $Q_{(\mathrm{q})}=\mathrm{A}_{(\mathrm{c})}+\mathrm{B}_{(\mathrm{c})} j$, then the complex eigen-values of $Q_{(\mathrm{q})}$ are the same as the eigen-values of $Q_{\text {e(c) }}$. Besides, if $A$ is one eigen-value of $Q_{\mathrm{e}(\mathrm{c})}$, then $\lambda^{*}$ will be another one. Therefore, every real eigen-values of $Q_{e(e)}$ occurs an even number of times. There are $n$ pairs of complex-conjugate eigen-values or dual real eigen-values of the complex matrix $Q_{\mathrm{e}(\mathrm{c})}$. so we can get $n$ complex eigen-values with nonnegative imagery part of the quaternion matrix $Q_{(q)}$. (A real eigen-value is a complex eigen-value with zero imagery part.)

Moreover, if $\vec{x}_{(c)}=\left[\vec{x}_{1(c)}^{T} \vdots \vec{x}_{2(c)}^{T}\right]^{T}$ is a eigen-vector of the complex matrix $Q_{e(c)}$ for $\lambda$, then $\vec{x}_{(q)}=\left[\vec{x}_{1(c)}\right]-\left[\vec{x}_{2(c)}^{*}\right] \cdot j$ is a eigen-vector of the quaternion matrix $Q_{\text {(q) }}$ for $\lambda$, where the superscript * represents the complex conjugate, $\vec{x}_{1(c)}$ and $\vec{x}_{2(c)}$ are $n^{*} 1$ complex column vectors, $\vec{x}_{(c)}$ is $2 n^{*} 1$ complex column vector, and $\ddot{x}_{(q)}$ is $n^{*} 1$ quaternion column vector.

Consequently, we can use the eigen-values and eigen-vectors of the equivalent complex matrix to calculate the eigen-values and eigen-vectors of the quaternion matrix.

\section{SINGULAR VALUE DECOMPOSITION OFA QUATERNION MATRIX}

In [5], the author has proved the existence of the polar de- composition of a quaternion matrix and this proof can be used for the existence of SVD. We review this existence in theorem 3 and give a theorem and a detailed algorithm to calculate the SVD of a quaternion matrix.

Theorem 3 : Existence of the SVD of a quaternion matrix

Let $Q_{(9)}$ be a $n^{*} n$ quaternion matrix with rank $\mathrm{r}$, then there exist two unitary quaternion matrices $U_{(q)}$ and $V_{(\mathrm{q})}$ such that $U_{\langle q\rangle}^{H} \cdot Q_{\langle q)} \cdot V_{(q)}=\left[\begin{array}{cc}\Lambda_{r} & 0 \\ 0 & 0\end{array}\right] \equiv \Lambda$, where $\Lambda_{\mathrm{t}}=\operatorname{diag}\left\{\lambda_{1}, \ldots, \Lambda_{\mathrm{t}}\right\}$ and $\lambda$ 's are the positive singular values of $Q_{(q)}\left({ }^{\mathrm{H}}\right.$ means the hermitian transpose)

The proof of this theorem can be found in [5].

Theorem 4: The meanings and relations between the SVD of a quaternion matrix and the SVD of its equivalent complex matrix

Let the SVD of a quaternion matrix and its equivalent complex matrix are $Q_{(q)}=U_{(q)} \cdot \Lambda \cdot V_{(q)}^{H}$ and $Q_{e(c)}=U_{(c)} \cdot \Lambda^{\prime} \cdot V_{(c)}^{H}$, respectively, then

$$
\begin{aligned}
& Q_{(q)} Q_{(q)}^{H}=U_{(q)} \cdot \Lambda^{2} \cdot U_{(q)}^{H}, Q_{(q)}^{H} Q_{(q)}=V_{(q)} \cdot \Lambda^{2} \cdot V_{(q)}^{H} \cdot \\
& Q_{e(c)} Q_{e(c)}^{H}=U_{(c)} \cdot\left(\Lambda^{2}\right)^{2} \cdot U_{(c)}^{H}, Q_{e(c)}^{H} Q_{e(c)}=V_{(c)} \cdot\left(\Lambda^{\prime}\right)^{2} \cdot V_{(c)}^{H} .
\end{aligned}
$$

Hence, the meanings of the SVD of a quaternion matrix are :

(1) $\wedge^{2}$ is the real eigen-value diagonal matrix of $Q_{\{q)} Q_{\{q\}}^{H}$ and $Q_{(q)}^{H} Q_{(q)}$.

(2) The column vectors of $U_{(q)}$ and $V_{(q)}$ are the eigen-vectors of $Q_{(q)} Q_{(q)}^{H}$ and $Q_{(q)}^{\prime i} Q_{(q)}$, respectively.

Since $Q_{\text {e(c) }}$ is the equivalent complex matrix of $Q_{(q)}$, then $Q_{e(r)} Q_{e(c)}^{H}$ and $Q_{e(c)}^{H} Q_{e(c)}$ are the equivalent complex matrix of $Q_{(q)} Q_{(q)}^{H}$ and $Q_{(q)}^{H} Q_{(q)}$ by theorem 1 . Hence, the relations between $\Lambda^{\prime}$ and $\wedge, U_{(\mathrm{c})}$ and $U_{(\mathrm{q})}, V_{(\mathrm{c})}$ and $V_{(\mathrm{q})}$ are :

(1) $\wedge^{2}$ and $\left(\wedge^{\prime}\right)^{2}$ are the real eigen-value matrix of $Q_{(q)} Q_{(q)}^{H}$ and $Q_{\varepsilon(c)} Q_{e(c)}^{H}$, respectively, so every element of $\wedge^{2}$ will occur twice in $\left(\Lambda^{\prime}\right)^{2}$. Hence, deleting the half duplicate singular values in $\Lambda^{\prime}$ will become $\Lambda$.

(2) The column vectors of $U_{(\mathrm{q})}$ and $U_{(\mathrm{c})}$ are the eigen-vectors of $Q_{(g)} Q_{(g)}^{H}$ and $Q_{e(c)} Q_{\mathrm{e} \mid c)}^{H}$, respectively, so we can use theorem 2 to calculate the column vectors of $U_{(\mathrm{q})}$ by the column vectors of $U_{(\mathrm{c}) \text {. }}$

(3) By the same reason and step as (2), we can calculate $V_{\text {(q) }}$ from $V_{(\mathrm{c})}$

However, for each eigen-value, it occurs twice in $\Lambda^{\prime}$, so there are two different column vectors of $U_{(\mathrm{c})}$ (or $V_{\text {(c) })}$ can be used to calculate the column vectors of $U_{(\mathrm{q})}$ (or $V_{(\mathrm{q})}$ ). We will have $2^{n}$ numbers of choice of the column vectors of $U_{(q)}$ and $V_{(q)}$. As a result, the $\mathrm{SVD}$ of $Q_{(q)}$ is not unique.

In this paper, we use the first corresponding eigen-vector to calculate the column vectors of $U_{(q)}$ and $V_{(q)}$.

Therefore, by means of the theorem 2 and 4 , the algorithm to calculate the SVD of a quaternion matrix $Q_{(\mathrm{q})}$ can be performed 
by the following steps :

Step 1 : Calculate the equivalent complex matrix $Q_{\text {e(c) }}$ by (4).

Step 2 : Calculate the SVD of the $Q_{\text {e(c) }}$ by the conventional SVD of a complex matrix. Assume $Q_{e(\mathrm{c})=} U_{(\mathrm{c})} \wedge^{\prime} V_{(\mathrm{c})}{ }^{\mathrm{H}}$.

Step 3 : The singular matrix of $Q_{(q)} \wedge$, can be calculated by theorem 4 and $\Lambda^{\prime}$, and the two unitary quaternion matrices of $Q_{(q)}, U_{(q)}$ and $V_{(q)}$, can be computed by theorem 2 , $4, U_{(\mathrm{c})}$ and $V_{(\mathrm{c})}$

Example 1: Compute the SVD of a quaternion matrix $Q_{(q)}$ $Q_{(q)}=\left[\begin{array}{cc}1+i+j+k & 2+i-k \\ 1-j+2 k & 3+2 j-2 j+k\end{array}\right]=\left[\begin{array}{cc}1+i & 2+i \\ 1 & 3+2 i\end{array}\right]+\left[\begin{array}{cc}1+i & -i \\ -1+2 i & -2+i\end{array}\right] \cdot j$

First, calculate the equivalent complex matrix and its SVD.

$$
\begin{aligned}
& Q_{\varepsilon(3)}=\left[\begin{array}{cccc}
1+i & 2+i & 1+i & -i \\
1 & 3+2 i & -1+2 i & -2+i \\
-1+i & -i & 1-i & 2-i \\
1+2 & 2+i & 1 & 3-2 i
\end{array}\right], \quad \Lambda^{\prime}=\left[\begin{array}{cccc}
5.75 & 0 & 0 & 0 \\
0 & 5.75 & 0 & 0 \\
0 & 0 & 0.91 & 0 \\
0 & 0 & 0 & 0.91
\end{array}\right]
\end{aligned}
$$

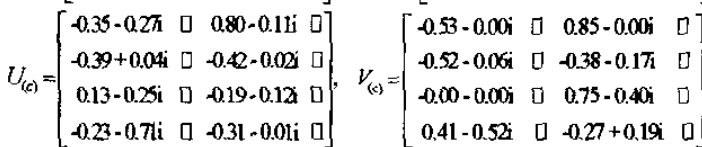

We only need half column vectors of $U_{(\mathrm{c})}$ and $V_{(\mathrm{c}) \text {, so the other }}$ half ones are not shown above. Therefore, the SVD of $Q_{(\mathrm{q})}$ are :

$$
\begin{aligned}
& \Lambda=\left[\begin{array}{ll}
575 & 0 \\
0 & 091
\end{array}\right], \quad U_{(\phi)}=\left[\begin{array}{cc}
035-027 i & 0.82-011 i \\
039+004 i & -0.28-002 i
\end{array}\right]+\left[\begin{array}{cc}
-0.13-025 i & 019-012 i \\
023-071 i & 031-001 i
\end{array}\right] j . \\
& V_{(q)}=\left[\begin{array}{cc}
-0.53-0.00 i & 0.85-0.00 i \\
-0.52-0.06 i & -0.38-0.17 i
\end{array}\right]+\left[\begin{array}{cc}
0.00-0.00 i & -0.55-0.00 i \\
-0.41-0.52 i & 0.27+0.19 i
\end{array}\right] j
\end{aligned}
$$

\section{APPLICATIONS OF THE SVD OF A QUATERNION MATRIX}

Recently, using a quaternion matrix $f_{(q)}(m, n)$ to represent a color image has been adopted by many researchers [7? [9]:

$$
f_{(q)}(m, n)=f_{R}(m, n) i+f_{G}(m, n) j+f_{B}(m, n) k
$$

, where $f_{R}(m, n), f_{G}(m, n), f_{B}(m, n)$ represent the R, G, B parts of the color image.

We also adopt this representation, then the SVD of a color image is

$$
f_{(q)}=U_{(q)} \cdot \Lambda \cdot V_{(q)}^{H}
$$

where $\bigwedge, U_{(q)}$ and $V_{(q)}$ can be computed by the steps in Sec. 3 .

From the SVD of a color image, many useful image processing methods by SVD can be extended to a color image without separating the color image into three channel images. Here, we will introduce some useful color image processing applications.

\section{Eigen-images :}

Similar to the SVD of a gray image [6], the SVD of a color image $f_{(\mathfrak{Q})}$ can be decomposed into the summation of vector outer products.

$$
f_{(q)}=U_{(q)} \cdot \Lambda \cdot V_{(q)}^{H}=\sum_{i=1}^{R} \lambda_{i} \cdot u_{i(q)} \cdot v_{i(q)}^{H}
$$

, where $u_{\mathrm{i}(q)}$ and $v_{\mathrm{i}(q)}$ are the column vectors of $U_{(q)}$ and $V_{(q)}$, respectively, $\lambda_{i}$ is the diagonal terms of real matrix $\Lambda$, and $R$ is the rank of $f_{(q)}$. Every product $u_{i(q)} v_{i(q)}^{H}$ is called an eigen-image. Hence, the color image $f_{(q)}$ can be considered as the linear combination of $R$ color eigen-images. Fig. 1 illustrates some color eigen-images of Mandrill baboon and Tiffany. (The sizes of these images is $256^{*} 256$ ). These figures illustrate normalized absolute-value versions of the first, second, and fourth eigen-images obtained from the decomposition of the original images. Similar to the complex matrix, the preceding eigen-images represent the low-frequency components of the original image, and the later ones represent the high-frequency components.

\section{Image Compression}

The singular values of the two color images in Fig. 1 are shown in Fig. 2. The same as the conventional complex case, the singular values decay very fast. Hence, an approximate representation of the color image can be formed by summing the preceding $\mathrm{K}$ eigen-images :

$$
\left[f_{(q)}\right]_{K}=\sum_{i=1}^{K} \lambda_{i} \cdot u_{i(q)} \cdot v_{i(q)}^{H} \quad(K \leq R)
$$

The real part of $\left.f_{(q)}\right]_{K}$ is small and will decrease to zero when $\mathrm{K}$ increases to $\mathrm{R}$ and one color image have only 3 components, so the real part of $\left.V_{(q)}\right\}_{K}$ will be discarded. However, the ignorance of the real part of $\left[f_{(q)}\right]_{K}$ is acceptable.

In general, small $K$ can provide a good estimation of original color image. Consequently, the storage requirements for this color image drop from $3 N^{2}$ to $K(2 * 4 N+1)$. ( $\mathrm{K}$ real singular values; $2 \mathrm{~K} N^{*} 1$ quaternion vectors. Besides, one quaternion vector is equivalent to four real vectors.) Fig. 3 illustrates three estimated images of the color images in Fig. 1 with $\mathrm{K}=10,20$ and 40.

Besides, to analyze the performance of this estimation, the PSNR of the estimated color images for $K=10,20,30,40,50$, 60 and for three well-known color images, "Baboon, Lena and Tiffany", are given in Table 1. The color image Baboon has many high frequency components, so the performance is the worst among these 3 images. The color image Tiffany has less high frequency components, so the performance is the best.

\section{Image Enhancement}

Image enhancement can be done simply by weighting the singular values. Similar to the conventional case, there are two kinds of image enhancement by means of SVD, linear weighting and nonlinear weighting [6].

The linear weighting is

$$
[f]_{(q)}=\sum_{i=1}^{R}(1+m \cdot i) \lambda_{i} \cdot u_{i(q)} \cdot v_{i(q)}^{H} \quad(m \geq 0)
$$

Linear weighting will enhances the high frequency components, so it is similar to a high pass filter. On the other hand, the nonlinear weighting is

$$
[f]_{(q)}=\sum_{i=1}^{R} \lambda_{i}^{\alpha} \cdot u_{i(q)} \cdot v_{i(q)}^{H}
$$

If $\alpha>1$, nonlinear weighting is a low pass filter. Contrarily, if $\alpha$ $<1$, it will be a high pass filter [6].

Fig. 4 illustrates the effects of the image enhancement. The enhanced color image of the linear weighting with $\mathrm{m}=0.005$ is shown in Fig. 4(a). The enhanced color images of the nonlinear weighting with $\alpha=1.1$ and 0.9 are shown in Fig. 4 (b) and (c), respectively. 


\section{CONCLUSION}

In this paper, we first review some important concepts of a quaternion matrix, such as left and right eigen-values, eigen-vectors, eigen-value class and the equivalent complex matrix etc. Then we discuss the SVD of a quaternion matrix and give an efficient algorithm to calculate it by the SVD of the equivalent complex matrix. Finally, we give some applications by using the SVD of a quaternion matrix.

The SVD of a quaternion matrix can be used in many areas. such as the inverse and pseudoinverse of a quaternion matrix and color image processing. By the SVD of a quaternion matrix, we can compute the SVD of a color image processing withou separating it into three channel images. Then, many useful image processing method by SVD can be extended for color images, such as eigen-images, image compression, image enhancement and denoise. Consequently, SVD of a quaternion matrix is a useful tool for color image processing

\section{REFERENCES}

[1] W. R. Hamilton, "Elements of Quaternions", Longmans, Green and Co., London, 1866

[2] H. C. Lee, 'Eigen-values and Canonical forms of matrices with quaternion coefficients", Proc. R.L.A. 52, Sect. A, 1949, pp 253-260

[3] J. L. Brenner, "Matrices of quaternions", Pacific J. Math. , 1951, pp. 329-335.

[4] A. Bunse-Gerstner, R. Byers and V. Mehrmann, "4 Quaternion QR Algorithm", Numerische Mathematik, Vol. 55, 1989 , pp. 83-95.

[5] F. Zhang. "Quaternions and matrices of Quaternions", Linear algebra and its applications, 1997, pp. 21.57.

[6] H. C. Andrews and C. L. Patterson, 'Singular Value Decomposition and Digital Image Processing', IEEE Trans. Acoustics, Speech, and Signal Processing. February 1976, pp. 81-108

[7] S. C. Pei, J. J. Ding, and J.H. Chang 'Efficient implementation of quaternion Fourier transform, convolution, and correlation by 2-D FFT', IEEE Trans. Signal Processing vol. 49, No.11, Nov. 2001. pp 2783-2797

[8] C. J. Evans, S. J. Sangwine and T. A. Ell, 'Color-sensitive edge detection using Hypercomplex filters'. Processing EUSIPCO 2000, Tampere, Finland, Sep. 2000.

[9] S. J. Sangwine and T. A. Ell, 'Hypercomplex auto- and cross-correlation of color images', p. 319-323. IEEE International Conference on Image Processing. 1999.

\begin{tabular}{|c|c|c|c|c|c|c|}
\hline $\mathrm{K}$ & 10 & 20 & 30 & 40 & 50 & 60 \\
\hline Baboon & 21.8925 & 23.3979 & 24.5634 & 25.6661 & 26.7536 & 27.8328 \\
\hline Lena & 23.2482 & 26.4791 & 28.6795 & 30.4901 & 32.1653 & 33.6997 \\
\hline Tiffany & 27.2786 & 30.7416 & 33.1189 & 35.0070 & 36.6453 & 38.1338 \\
\hline
\end{tabular}

Table 1. PSNR of the approximated color images, Baboon, Lena and Tiffany, with different $K$ eigen-images.
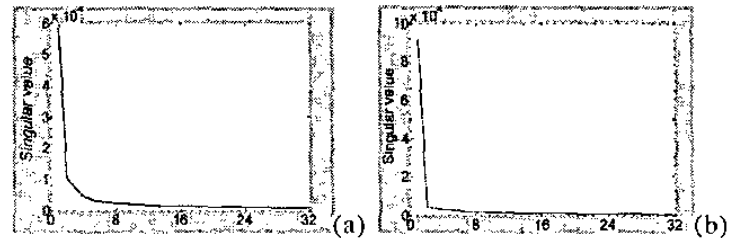

Fig. 2. Singular values of two color images. (a) Mandrill baboon.

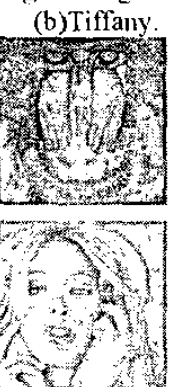

(a)
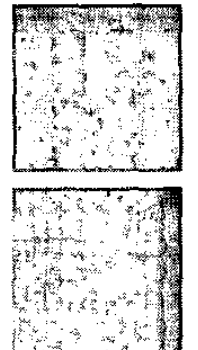

(b)
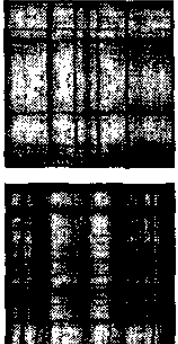

(c)
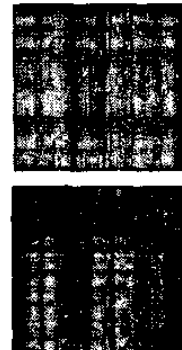

(d)

Fig. 1 Selected eigen-images of Mandrill baboon and Tifrany. (a) original image. (b) $u_{i(q)} v^{H}$

(c) $u_{z(q)} v_{z(q)}^{H}$

(4) $u_{4(q)} v_{4(q)}^{H}$
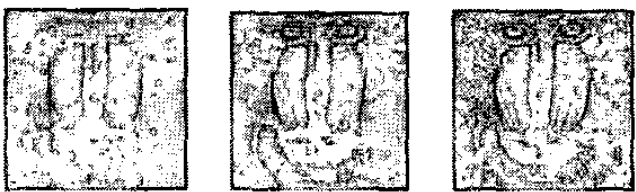

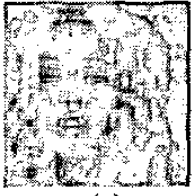

(a)

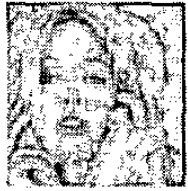

(b)

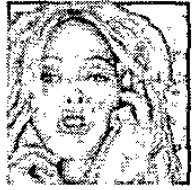

(c)
Fig. 3. Selected Partial sums of Mandrill baboon and Tiffany. (a) $\left[f_{10}\right]_{(q)}$. (b) $\left[f_{20}\right]_{(q)}$. (c) $\left[f_{40}\right]_{(q)}$.
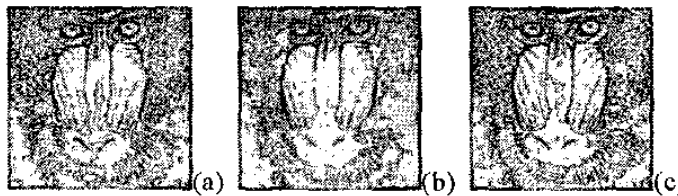

Fig. 4. Image Enhancement by linear and nonlinear weighting. (a) Linear weighting with $\mathrm{m}=0.005$. (b)(c) Nonlinear weighting witha $=1.1$ and 0.9 , respectively. 